\title{
Hybrid Differential Evolution Based Probabilistic Model to Support Quality Aware Best Path Routing Scheme for Mobile Adhoc Networks
}

\author{
Priya, P. Suganthi
}

\begin{abstract}
The Mobile Ad hoc Networks (MANETs) today represent a new system containing wireless mobile nodes to dynamically and freely organize the topologies of the network without having any communication infrastructure. Owing to the traits such as temporary topology and the absence of a proper centralized authority routing can be a very important issue faced by the ad hoc networks. This multipath routing algorithm has established various paths between the source node and its destination node thus spreading traffic load along various routes. This will be able to alleviate congestion of traffic on a particular path. Thus, the multipath routing algorithms were able to provide route resilience that ensured data transmission reliability. For the purpose of this work, there was a multipath routing scheme which is called the Ad hoc On-demand Multipath Distance Vector Routing (AOMDV) Protocol. This was proposed by means of employing a hybrid Fire Fly $(F F)$ with Differential Evolution (DE) algorithm. This approach was able to achieve better, as well as, reciprocal advantages in a dynamic and hostile network situation. Thus, the proposed scheme of routing was a very powerful method to find effective solutions to MANET routing problems. The proposed method is a well-known probabilistic metaheuristic algorithm to improve the quality aware best path routing protocol. Simulation results indicate that the proposed method FF-DE achieves better performance than $A O M D V$ and $F F$.
\end{abstract}

Keywords : Ad hoc On-demand Multipath Distance Vector Routing (AOMDV), Differential Evolution (DE) Algorithm, Fire Fly (FF) Algorithm and Mobile Ad Hoc Networks (MANETs).

\section{INTRODUCTION}

The Mobile Ad hoc Network (MANET) can be an interconnection of the autonomous mobile nodes that is by the wireless links that form a dynamic topology. This provides multi-hop communications that do not use physical network infrastructures like centralized administration, cables, access points, servers and routers. Every mobile node will act as a router and as a node. The network property is highly desirable in marine communications, home, industry, marine communications, aircraft, and war zones. The MANET issues were [1]: (i) an unpredictable set of link properties exposing packet collision with signal propagation, (ii) mobility of node that creates dynamic topology, (iii) a limited battery life for mobile devices, (iv) both hidden and

Revised Manuscript Received on December 5, 2019.

* Priya: Research scholar, Department of computer science,NKR government Arts college for Women, Namakkal, Tamilnadu, India

Dr.P.Suganthi: Head and Assitant Professor, Department of Computer Science, N.K.R.Government Arts College for Women, Namakkal, Tamilnadu, India, Email: mahadhimohit@gmail.com. exposed problems that occur at the time the signals of both nodes collide with one another. (v) route maintenance that can be quite challenging owing to the changing behaviour of that of the medium of communication, and (vi) lack of security of the boundaries of the MANET that results in attacks such as Denial of Service (DoS), message contamination, message replay, data tampering, leakage of information, active interfering and passive eavesdropping. Among these, routing is the most critical.

There is a very significant role played by network performance in the MANETs for the provision of quality of services. There was an absolute algorithm of routing that was an attempt at finding optimal paths for the successful transmission of data. There have been various algorithms striving to identify optimum paths which are the Dijkstra algorithm, breadth-first search, and bellman ford algorithm. All these solve the problem of the optimum path in the polynomial time which is useful for the fixed infrastructure that is either wired or wireless. In the case of a dynamic wireless network, the algorithms These entire algorithms solve the optimum path problems within the polynomial time. For the dynamic networks, the results of the algorithm will be unexpectedly high in terms of computational complexities and this was for real-time communication. For the purpose of transmission of data, the routing algorithms were grouped into three: the reactive, the proactive and the hybrid protocols. The proceeds with various types of methodology will normally maintain the routing table as a proactive protocol. At the same time, the reactive protocol will upgrade the routing table at the time there is a need for communication [2].

The hybrid approaches, on the other hand, combine all the features. Owing to the frequent changes in the topology of the network, the proactive approaches were not found to be suitable for situations of multipath routing. Thus, the on-demand approaches have been preferred for the MANET multipath routing. Recently, these multipath routing approaches have been introduced for overcoming all limitations of single-path routing.

There are multiple routes identified between the source and the destination. The multipath approaches are beneficial in many ways like better bandwidth utilization, higher network lifetime, higher throughput and lower end-to-end delay. This is applied to load balancing to carry out traffic through various paths. It also decreases the congestion of the network and protects against any route failure [3]

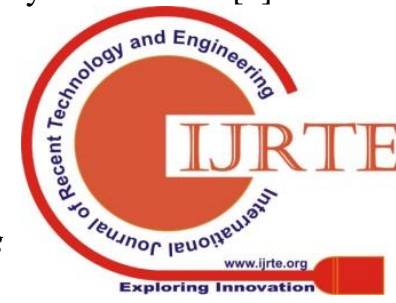


Sometimes these protocols may be enough for a particular class of applications of the MANET. In certain cases, however, they may not be sufficient for supporting more demanding applications like video or audio. These applications will need the network to be able to provide a certain guarantee on the achieved Quality of Service (QoS). This can be achieved by a particular mechanism like the QoS routing in order to identify the route which is the best that can satisfy the needs in the best manner. The QoS routing seems to be a solution that handles all these problems. It has the need to find a new route from the source to the destination which should be able to satisfy an end-to-end requirement. This can be in the form of loss probability, delay or bandwidth. The QoS is challenging to achieve in the case of ad hoc networks compared to the wired networks. The QoS refers to a set of requirements of service that is met by a network at the time of transporting a flow. The flow as a packet stream from the source to its destination that has a QoS which is associated. The fundamental need for a mechanism of the QoS will be measurable as a performance metric. There are some typical metrics of the QoS that are the bandwidth available, path reliability, hop count, packet jitter, and rate of packet loss. The primary issue in providing a QoS guarantee is the manner in which the paths are determined to satisfy the constraints and solving this problem is called QoS aware routing [4].

An Ad Hoc On-Demand Distance Vector (AODV) routing protocol can bring down the actual number of broadcasts required by means of creating the routes based on demand. The AODV authors had classified this to be a pure on-demand acquisition system as the nodes not found on the chosen path cannot maintain any routing information or can participate in the exchange of routing tables. The process of route discovery was initiated at the time the source was not a valid one. The broadcast messages were relayed until such time the destination or its intermediate node was sufficiently fresh in terms of route to its destination. For the purpose of ensuring loop freedom and freshness of path, the AODV makes use of sequence numbers. The intermediate nodes will be able to reply to a Route Request (RREQ) in case they have a route to their destination and if the corresponding sequence number was higher than equal to the one in the interest. The duplicate RREQs are discarded. The request received is cached for a particular time for establishing a reverse path. During the occurrence of any link failure, its immediate upstream node will inform the upstream neighbours to the source node. The source node can be decided to re-initiate the route discovery in case the route is still required. For the final note, it will have to be mentioned that the periodic HELLO message used for maintaining the node and its local connectivity. This happens for a considerable amount of control overheads [5].

The techniques of swarm optimization were used in the MANET to find an optimal solution from all possible outcomes. There had been some diverse techniques of optimization with better performance rendering better results. The primary concern was the focus that was on the approaches to optimization making the network more efficient and reliable. By using these techniques of optimization, it may be easier to identify the eligible solutions for the outcomes. There were many approaches to optimization that were used for identifying the best and most optimal solutions. There were algorithms that were inspired biologically to identify them. This referred to the performance of nature. Using these diverse algorithms, the problems were solved. The advantages of this were: Firstly, reaching a solution for which no mathematical approaches had to be followed. Secondly, the results were accurate and quick 6].

The DE had been proposed in the year 1996 by Storn and Price. The idea behind this was to be able to retrieve the solution that was best suited. For solving a problem in optimization, of an optimization problem. Actually, to solve any problem of optimization, there is a need to identify a factor variable based on which the entire system will deviate. The choice of a suitable result is best suited to the solution. The variables were framed into an objective function and the function value that was computed if the solution was the best. The DE algorithm works in four different steps: initialization, mutation, crossover and finally selection. Initialization here takes place one time and the remaining three are in an iterative manner until the condition for termination is met. The DE will own two of the control parameters ( $\mathrm{F}$ and $\mathrm{Cr}$ ) that affect their results. The parameters will get tuned in accordance with the application and their values are generated using a self-adaptive strategy. For the MANETs, searching a secure path for data dissemination is a hectic process as it may be vulnerable to certain attacks. The DE can be a simple mathematical model that has a complex process that is evolutionary and can facilitate searching for a secure data dissemination path [7].

The FF algorithm is a population-based optimization algorithm which finds the global optimal for the given objective functions. In this work, hybridization of the FF with $\mathrm{DE}$ algorithm is proposed for routing in MANET. The remaining part of the investigation is organized into the following sections. Section two discusses related works in literature. Section three explains various methods used in the work. Section four discusses experimental results and section five concludes the work.

\section{RELATED WORKS}

Chaudhry et al., [8] had made a new proposal for an Adaptively modified PSO (APSO) to fit into this scenario and also had reinforced this by using the Forwarding Search Space (FSS) technique for the purpose of overcoming the convergence of the PSO and the time taken for computation of all related issues. This was able to significantly improve PSO performance. In the FSS, the Forwarding Zone (FZ) was chosen between the source and the destination.

The optimal solution was within this area and the APSO was applied to be an effective mode of routing in the FZ area as opposed to a complete network.

For making use of the other complementary traits of the APSO and FSS, there was a hybrid FZ-APSO that was proposed for the purpose of routing in a dense network. This has minimum delay and consumption of energy for increasing network lifetime. 
Labed et al., [9] had made a proposal of a new routing protocol that was based on the Ant Colony Optimisation (ACO) hybridization and a 2-opt heuristic along with the optimisation of the parameters of the ACO. With the vast scope of these parameters, the Genetic Algorithm (GA) was used for minimising the problem's complexity. The method's implementation was realised using MATLAB. For validating these results based on the parameters of the QoS (the normalized overhead load, throughput, and end-to-end delay) there was a comparison made with the AOMDV routing protocol.

Jaiswal and Kaur [10] had proposed the AOMDV along with a Fitness Function (FF-AOMDV) and the Dragonfly topology. This fitness function was employed to identify the best path from supply to its destination for reducing the consumption of energy of multipath routing by means of using the Dragonfly topology. The proposed FF-AOMDV protocol along with the dragonfly topology had been evaluated in terms of performance with the Network simulator Version 2 (NS-2). A comparison of this protocol with the AOMDV and the Ad-hoc on Demand Multipath Routing with Life Maximization (AOMRLM), two of the most popular protocols in the area was made. The results demonstrated that the FFAOMDV proposed with the Dragonfly topology was able to outperform both the AOMDV and the AOMR-LM in most parameters and metrics.

The DE was applied by Prabha and Yadav [11] for solving issues in the optimal path for transmission of data from the source to the destination in the MANETs. This model based on DE makes use of the analysis to establish the optimal path that is best available in the MANETs. The cost of transmission is considered in searching for the consumption path. This DE is used in the form of a search tool to look out for a minimum transmission consumption by means of an established model. In the final stage, the results that were acquired by the algorithm proved the effectiveness of the protocol compared to the GA and PSO.

In the recent few years, there have been several algorithms that were formulated for the multicast problem to be in the form of a single-objective problem. Wei et al., [12] had made a proposal of another Multi-Objective Differential Evolution algorithm that was known as the MOMR-DE for resolving the problem of Multicast Routing. For the MOMR-DE, the bandwidth, jitter, delay, cost and network lifetime were the five main objectives. Also, there were three other QoS constraints that permitted delay, minimum requested bandwidth and minimum allowed jitter that was included. Additionally, this modified both mutation and crossover operators in building the shortest path to the multicast tree for maximizing the lifetime of the network and for minimizing jitter, delay, and cost. The results of simulation proved the method to achieve convergence that was faster and was more preferred for a MANET multicast routing.

Vijayalakshmi and Rao [13] had made a proposal of another Enhanced Differential Evolution Cuckoo Search routing protocol (EDE-CS) for construction of a consistent multicasting communication. This network was a mixture of two different algorithms of optimization which were the Cuckoo Search and DE. Clustering was made for reliable packet transmission with the DE and the CS to identify the best path for routing and transmitting messages. NS2 was a tool for a simulation that gives the proposed method's consequence by means of comparing it with the current protocol and was illustrated it with them is a simulation tool that gives the consequence of the proposed method by comparing it with the existing protocol. So, it obviously illustrates that the proposed EDE-CS protocol was effective in comparison with the current ones.

Another effective scheme of routing for the transmission of messages to various nodes is called Multicast routing. These applications will need several QoS guarantees that need to be satisfied. Another Genetic-oriented QoS Multicast Routing (GA-QMR) algorithm was proposed by Singh et al., [14]. The algorithm proposed was to efficiently identify an optimal multicast tree that satisfies various parameters of the QoS compared to the other algorithms. This algorithm will have the capacity of exploring various paths from the node and choose the best one on the basis of the parameters and their quality to achieve global convergence. These routes will have to meet the bandwidth, rate of packet success, jitter, and end-to-end delay.

Kondaiah and Sathyanarayana [15] had introduced another new scheme for intrusion detection to establish a path that was secure, and this made use of the genetic neuro-fuzzy system. The paths existing between the source and destination were generated. The work aimed at identifying the paths that were secure using the Self Adaptive Firefly based PSO (SA-FPSO) Algorithm. In the final stages, all secured paths were generated for data communication. The results simulation proved that the SA-FPSO scheme had outclassed all current work using values of $0.04319 \mathrm{sec}$, 0.691 , and 0.769 that was for the delay, rate of detection and throughput.

\section{METHODOLOGY}

Owing to the dynamic nature of the MANET, routing can be quite challenging. Link breakages and node failures can result in loss of resources. The basic issue that arises in the MANETs was the choice of an optimal path between the nodes. For data packet routing there were many different schemes that were proposed like the Dynamic Source Routing (DSR) and the AODV. These routing protocols made use of the minimum hop count or the path that was the shortest to be its primary metric for the selection of paths [16]. For the purpose of this section, the multipath routing protocol with the FF Algorithm, the DE, the hybrid FF-DE algorithm and the AOMDV that were discussed. 


\section{A. Ad hoc On-demand Multipath Distance Vector Routing (AOMDV) Protocol}

The AOMDV was an extension of the AODV to compute several loop-free and link-disjoint paths. For keeping track of several routes, routing entries for all destinations will consist of a list of the subsequent hops with their hop counts. All next-hops will have one sequence number. For every such destination, the node will maintain an advertised hop count that was defined as a maximum hop count which was used for forwarding route advertisements to the destination. Every duplicate route advertisement that was received by the node will be able to define a new and alternate path to its destination. For ensuring its loop freedom, the node may accept another path to its destination. This will be if it has a hop count that is lower than the one advertised for the destination. Since the maximum hop count is employed here, its advertised hop count may not change. At the time a route advertisement has been received for a particular destination with a higher sequence number, the advertised hop count and hop list will be reinitialized [17].

The AOMDV may be used to identify a node-disjoint or the link-disjoint routes. In order to find a node-disjoint route, every node will not immediately deject the duplicate RREQs. Every RREQ that arrives through another neighbour of the same source will define another node-disjoint path. This is owing to the fact that the nodes are not able to broadcast a duplicate RREQ so that any of the RREQs in the intermediate node will not traverse in the same node. To ensure multiple link disjoint routes are got, the destination will reply to the RREQs through their unique neighbours. Once the first hop is complete, the RREPs will follow reverse paths that are link-disjoint. The trajectories for every RREP will intersect at the intermediate node and take another reverse path to its source [18].

The primary advantages of the AOMDV were the establishment of the route on-demand are as follows. It creates loop-free nodes. It maintains connectivity. It recovers from failure quickly and efficiently. The disadvantages of the AOMDV were: it has more overheads of the message at the time of route discovery owing to increased flooding. The destination will reply to multiple RREQs and this leads to lower overhead packet which were in response to one single RREQ packet resulting in a heavy control overhead.

\section{B. Fire Fly (FF) Algorithm}

The FF algorithm, on the other hand, was developed by Yang 2008, Yang 2009 which was based on the flashing traits of fireflies. For purposes of simplicity, it idealises these flashing traits as below [19]:

- All fireflies are assumed to be unisex and so they are attracted to that of the other fireflies irrespective of their sex;

- The attractiveness of the fireflies was proportional to that of their brightness and so for any of the two flashing fireflies, the one that is less in terms of brightness moves towards the one that was brighter. As their attractiveness is proportional to their brightness, they decrease when distance increases. If there is no brighter one, the firefly moves randomly;
- The light intensity or brightness of the firefly will be affected and determined by the objective function landscape that is optimized.

For the FF algorithm, two important issues are considered: the light intensity will vary based on attractiveness. For purposes of simplicity, it may be assumed that the actual attractiveness of the FF will be determined by the light intensity and this, in turn, is connected to the encoded function. If the brightness I for an FF at a certain location $\mathrm{x}$ is chosen to be $\mathrm{I}(\mathrm{x}) \alpha \mathrm{f}(\mathrm{x})$, the attractiveness $\beta$ will be relative and will be seen as according to the eyes of the beholder. It varies with the actual distance $\mathrm{r}_{\mathrm{ij}}$ between the FF $i$ and the FF j. With a decrease in light intensity, it is absorbed in the media letting the attractiveness vary with absorption. As per the inverse square law [20], light intensity (I (r)) at distance $r$ from the light source $\left(1_{s}\right)$ may be computed as per equation (1):

$$
I(r)=l_{s} / r^{2}
$$

Here, light will be absorbed using a constant coefficient of light absorption $(\gamma) \in[0, \infty]$ and the equation can be formed as in the Gaussian using equation (2):

$$
\beta(r)=\beta_{0} e^{-\gamma r^{2}}
$$

$\beta(r)$ is the attractiveness of an FF at distance $r,{ }^{\beta}$ the attractiveness when $r=0$.

It can be assumed that both $i$ and $j$ are the two fireflies with positions $X_{i}\left(x_{i}, y_{i}\right)$ and $X_{j}\left(x_{j}, y_{j}\right)$, respectively. The distance $\left(r_{i j}\right)$ between both fireflies was computed based on the Euclidean by equation (3):

$$
r_{i j}=\left\|X_{i}-X_{j}\right\|=\sqrt{\left(x_{i}-x_{j}\right)^{2}-\left(y_{i}-y_{j}\right)^{2}}
$$

So, the one that is less bright with $i$ 's new position $\left(\mathrm{X}_{\mathrm{i}}\right)$ and the movement towards a more attractive $\mathrm{FF} j$ is computed using equation (4):

$$
X_{i}=X_{i}+\beta_{0} e^{-\gamma r_{i j}^{2}}\left(X_{j}-X_{i}\right)+\alpha \epsilon_{i}
$$

In (4) $\in_{i}$ is a random variable vector, and $(\alpha) \in[0,1]$ the randomization parameter..

\section{Differential Evolution (DE) Algorithm}

The performance of the DE is considered to be superior in solving issues that were exposed in the year 1996, in the IEEE international evolutionary computation conference. It was based on the individual differences in utilizing random research within the solution space. It also made use of "selection", "recombination", and "mutation" for computing. It used the available information between differences that result in search and this may be unstable. The $\mathrm{DE}$ is quite similar to the GA and the process of the DE is as below [21]:

Initialization: Setting up of parameters and initializing the Target Vector. 


$$
X_{j, i, 0}=\operatorname{rand}_{j}(0,1) *\left(b_{j, U}-b_{j, L}\right)+b_{j, L}
$$

Mutation: All common DE strategies are by a mutation vector formula for producing change. They are as in table 2 . The symbol of this strategy is DE/ $/ y$. Here, $x$ denotes the variance vector and $y$ the number of the variance vector.

In the $\mathrm{DE} / \mathrm{rand} / 1$

$$
V_{i, G+1}=X_{r 1, G}+F\left(X_{r 2, G}-X_{r 3, G}\right)
$$

For extension of search space, three vectors are chosen randomly that are $X_{r 1, G}, X_{r 2, G}$, and $X_{r 3, G}$ with equation (4) that obtains the Donor Vector as $V_{i, G+1}$.

For the DE/best/1

$$
V_{i, G+1}=X_{\text {best }, G}+F\left(X_{r 2, G}-X_{r 3, G}\right)
$$

Based on the DE/rand/1, the strategy uses its present best particle $X_{b e s t, G}$ for $X_{r 1, G}$

Recombination: A change is made to information by the donor vector with the target vector. The new "trial vector" known as $u_{i, G+1}$ and is generated after recombination. With the formulation below, it is decided if iteration $\mathrm{j}$ of component $\mathrm{i}$ can be composed using target vector xi or the donor vector vi.

$$
u_{j, i, G+1}=\left\{\begin{array}{c}
V_{j, i, G+1} \text { if rand } \leq C R \\
x_{j, i, G} \text { if rand }>C R
\end{array}\right\}
$$

Selection: Once this is completed it is compared to the Trial Vector and the Target Vector to choose the vector that is reversed to the subsequent generation.

$$
X_{i, G+1}= \begin{cases}u_{i, G+1} \text { if } F\left(u_{i, G+1}\right) \leq F\left(X_{i, G}\right) \\ X_{i, G} \text { otherwise }\end{cases}
$$

This includes the following steps:

Step 1 : Initialization.

Step 2 : Application of formula (5) to choose the Target Vector $\mathrm{X}_{\mathrm{i}, \mathrm{G}}$.

Step 3 : Evaluation of fitness.

Step 4 : Mutation: Three vectors are chosen in accordance with formula (6) or (7) for mutating in order to generate a Donor Vector.

Step 5: Recombination: The information between the Target and the Donor Vector is changed using formula (8) and thereby obtain its Trial Vector.

Step 6 : The Target Vector and the Trial Vector are compared and chosen by the fitness to identify the reserve to be taken to the subsequent generation.

Step 7 : If termination condition is not met go to Step 2 or output a solution

\section{Proposed Hybrid Fire Fly (FF) Algorithm with Differential Evolution (DE) Algorithm (FF-DE)}

The FF and the DE algorithm are both advantageous and work well for a wider range of problems of optimization. Here, a new hybrid algorithm that was based on the FF and the DE was proposed and this was a combination of the advantages of both, which was the attracting mechanism of that of the FF and the mixing capacity of the DE. This increases the diversity and convergence of the population. The primary difference between the FF and the DE is the manner in which new individuals were generated and used for each of the iterations [22].

From among the components of the algorithm, both intensification and diversification (exploitation and exploration) are the components of the meta-heuristic algorithm. For exploring the search space globally, the metaheuristic algorithms will have to generate a range of solutions. The strategy of intensification or exploitation will guide the individual to search within a local region and this is found at the time of search which can be a good solution in the region. The accuracy of the solution of the algorithm and the rate of convergence is enhanced by means of balancing both intensification and diversification.

Firstly, for all the earlier observations or investigations found in the literature showed that the FF was able to subdivide the entire population in terms of the mechanism of attraction through the light intensity variation. A variant of the FF will be able to escape from its local minima because of the mobility of the Lévy flight. These advantages further mean that the FF is good in terms of exploration and diversification. Also, owing to the efficiency of that of the mutation operator and the crossover operator, differential evolution may be getting better diversity. The DE also carries out a local search to improve both exploitation and exploration.

Additionally, updating its current local best in the entire population will ensure that the solutions are able to converge to the optimum. Diversification through mixing or regrouping of the entire population permits the algorithm to escape from its local optima. The mix and the regrouping of the individual location information will be obtained once the main iteration of that of the parallel FF and DE processes are made. The superiority of this mechanism of mixing and regrouping was to ensure the search focuses on the locations which are in the areas that are obtained in the previous phase as opposed to search or re-search the regions that are less promising. On the basis of these descriptions, the basic steps of the hybrid FF-DE are summarized as per the pseudo-code below. The parallel use of the FF and the DE will strike a proper balance between both exploration, as well as exploitation, for the entire process.

The Fig 1 shows the flowchart for hybrid FF-DE algorithm [23]. 


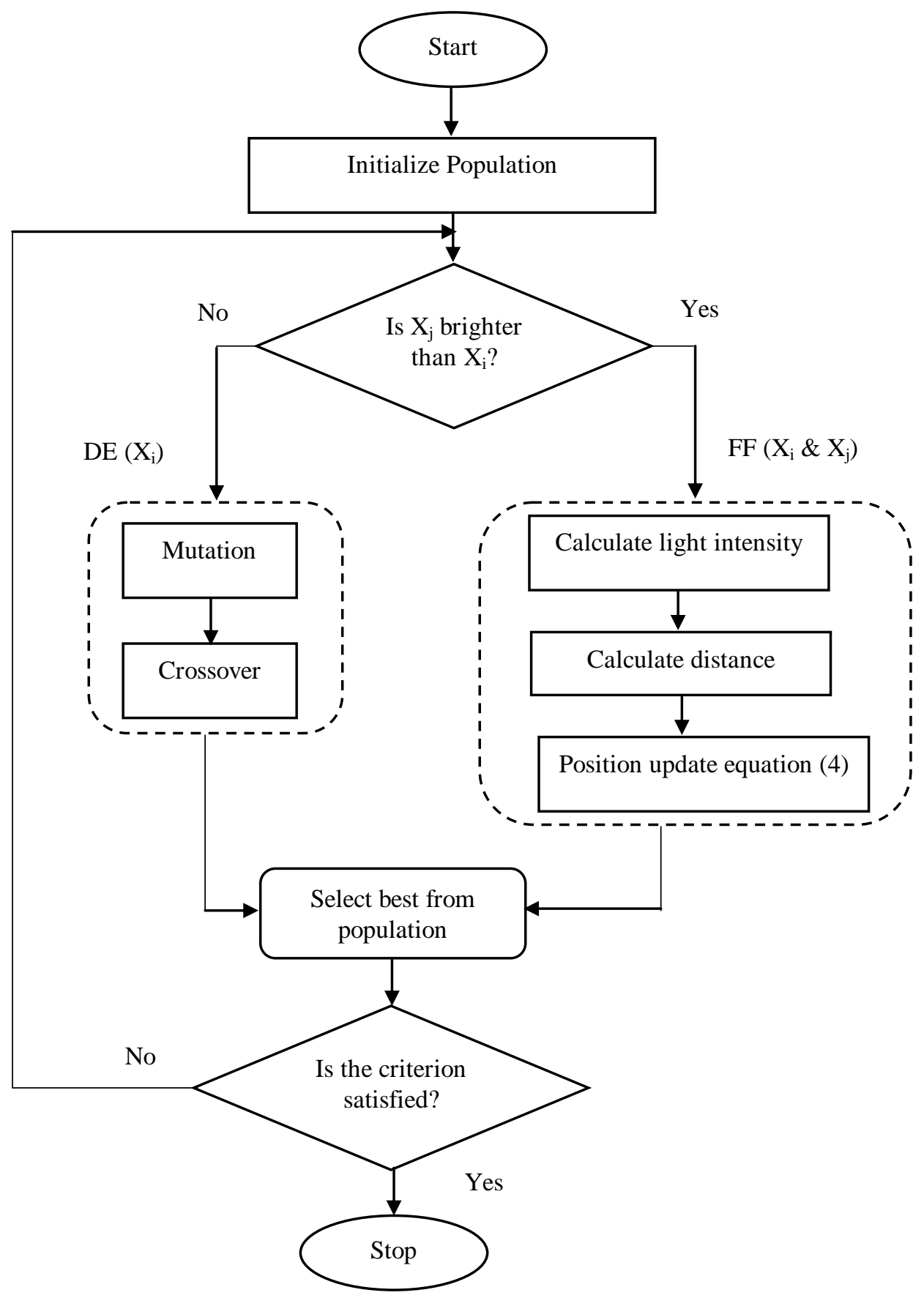

Fig 1 Flowchart for Hybrid FF-DE Algorithm

\section{RESULTS AND DISCUSSION}

In this section, the AOMDV, FF and FF-DE methods are used. Experiments are carried out using 100 to 600 number of nodes. The average Packet Delivery Ratio (PDR), average end to end delay, average number of hops to sink, jitter and normalized routing load as shown in Tables $\mathrm{I}$ to $\mathrm{V}$ and Figures 2 to 6.
Table I Average Packet Delivery Ratio for FF-DE

\begin{tabular}{|c|c|c|c|}
\hline Number of nodes & $\mathrm{V}^{\text {AOMD }}$ & FF & FF-DE \\
\hline 100 & 0.8237 & $1^{0.836}$ & 0.8917 \\
\hline 200 & 0.7793 & $\begin{array}{ll} & 0.791 \\
7 & \\
\end{array}$ & 0.8595 \\
\hline 300 & 0.7723 & 0.786 & 0.8307 \\
\hline 400 & 0.7264 & $5^{0.735}$ & 0.8013 \\
\hline
\end{tabular}




\begin{tabular}{|c|c|c|c|}
\hline 500 & 0.6823 & $7^{0.690}$ & 0.7449 \\
\hline 600 & 0.6518 & $9^{0.662}$ & 0.7062 \\
\hline
\end{tabular}

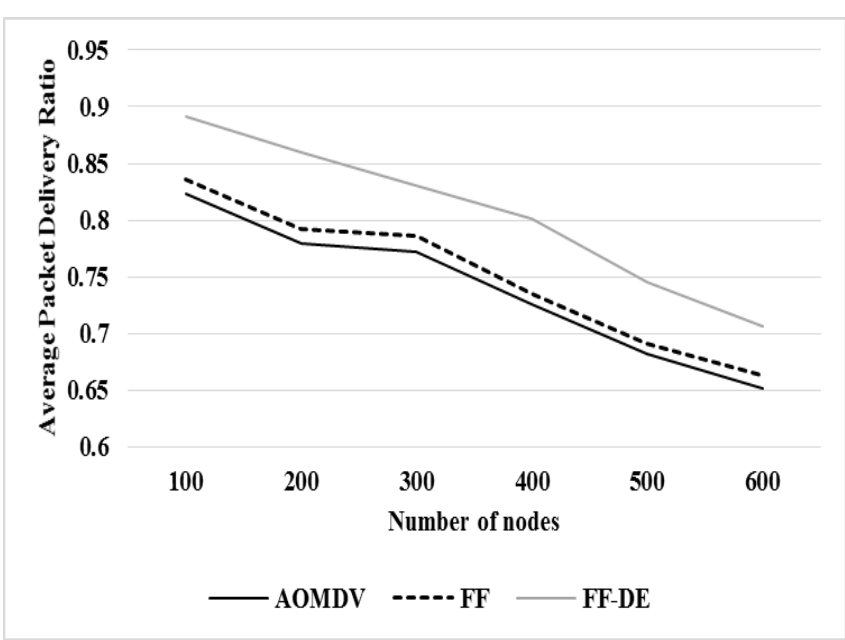

Fig 2 Average Packet Delivery Ratio for FF-DE

From the Fig 2, it can be observed that the FF-DE has higher average PDR by $7.92 \% \& 6.43 \%$ for 100 number of nodes, by $9.78 \% \& 8.21 \%$ for 200 number of nodes, by $7.28 \%$ $\& 5.52 \%$ for 300 number of nodes, by $9.8 \% \& 8.56 \%$ for 400 number of nodes, by $8.77 \%$ \& $7.55 \%$ for 500 number of nodes and by $8.01 \% \& 6.32 \%$ for 600 number of nodes when compared with AOMDV and FF.

Table II Average End to End Delay for FF-DE

\begin{tabular}{|l|l|l|l|}
\hline Number of nodes & AOMDV & FF & FF-DE \\
\hline 100 & 0.00172 & 0.00165 & 0.00164 \\
\hline 200 & 0.00211 & 0.00202 & 0.00203 \\
\hline 300 & 0.00438 & 0.00423 & 0.00411 \\
\hline 400 & 0.00837 & 0.00799 & 0.00795 \\
\hline 500 & 0.01535 & 0.01471 & 0.01415 \\
\hline 600 & 0.02478 & 0.02365 & 0.0236 \\
\hline
\end{tabular}

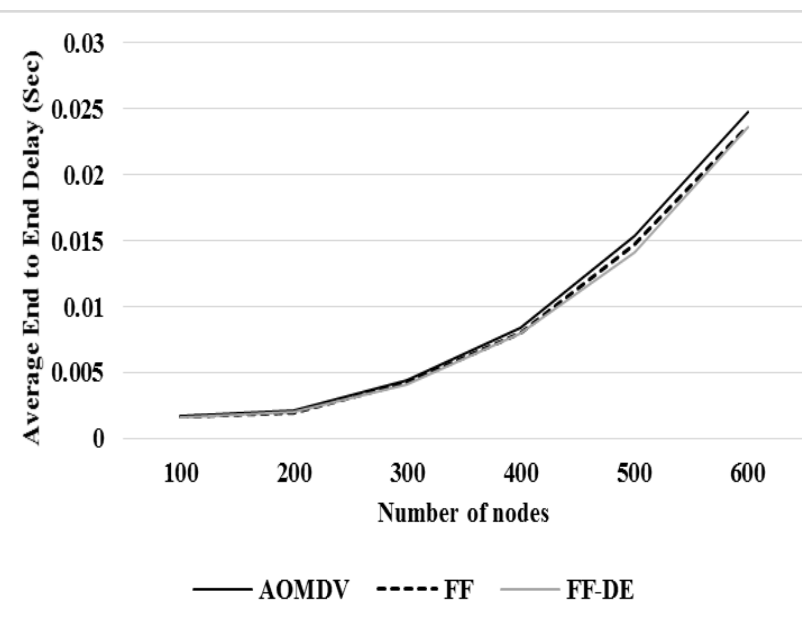

Fig 3 Average End to End Delay for FF-DE

From the Fig 3, it can be observed that the FF-DE has lower average end to end delay by $4.76 \% \& 0.61 \%$ for 100 number of nodes, by $3.86 \% \& 0.49 \%$ for 200 number of nodes, by $6.36 \% \& 2.87 \%$ for 300 number of nodes, by $5.14 \%$ $\& 0.5 \%$ for 400 number of nodes, by $8.13 \% \& 3.88 \%$ for 500 number of nodes and by $4.87 \% \& 0.21 \%$ for 600 number of nodes when compared with AOMDV and FF.

Table III Average Number of Hops to Sink for FF-DE

\begin{tabular}{|l|l|l|c|}
\hline Number of nodes & AOMDV & FF & FF-DE \\
\hline 100 & 6.1 & 5.8 & 5.9 \\
\hline 200 & 8.1 & 7.6 & 7.8 \\
\hline 300 & 9 & 8.3 & 8.7 \\
\hline 400 & 9.7 & 8.9 & 9.4 \\
\hline 500 & 9.8 & 9.2 & 9.5 \\
\hline 600 & 10 & 9.4 & 9.7 \\
\hline
\end{tabular}

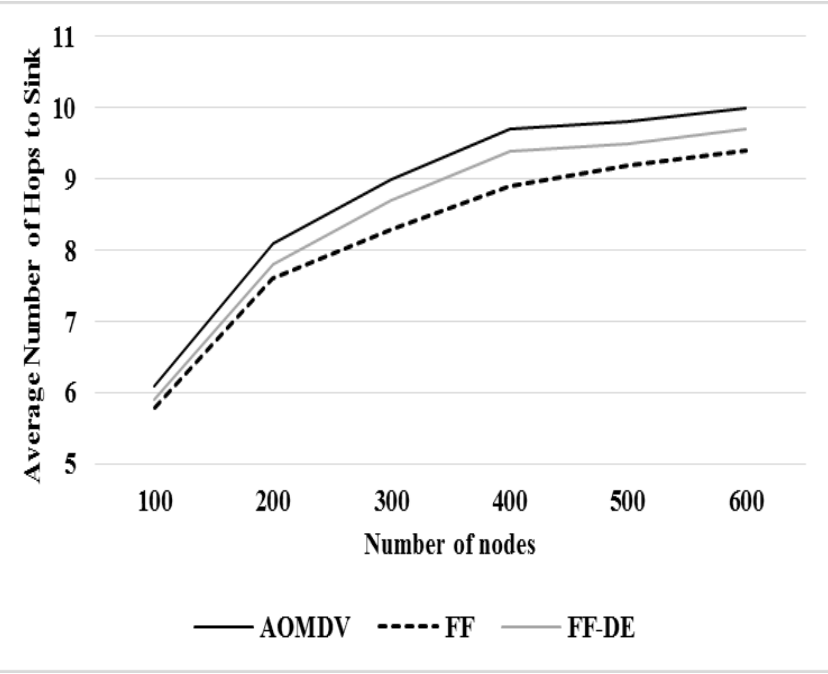

Fig 4 Average Number of Hops to Sink for FF-DE

From the Fig 4, it can be observed that the FF-DE has lower average number of hops to sink by $3.33 \%$ for 100 number of nodes, by $3.77 \%$ for 200 number of nodes, by $3.38 \%$ for 300 number of nodes, by $3.14 \%$ for 400 number of nodes, by $3.1 \%$ for 500 number of nodes and by $3.04 \%$ for 600 number of nodes when compared with AOMDV. The FF-DE has higher average number of hops to sink by $1.7 \%$ for 100 number of nodes, by $2.59 \%$ for 200 number of nodes, by $4.7 \%$ for 300 number of nodes, by $5.46 \%$ for 400 number of nodes, by $3.2 \%$ for 500 number of nodes and by $3.14 \%$ for 600 number of nodes when compared with FF.

Table IV Jitter for FF-DE

\begin{tabular}{|c|c|c|c|}
\hline Number of nodes & $\mathrm{V}^{\text {AOMD }}$ & $\mathrm{FF}$ & FF-DE \\
\hline 100 & $7^{0.0007}$ & $74^{0.000}$ & $6^{0.0007}$ \\
\hline 200 & $3^{0.0018}$ & $77^{0.001}$ & $2^{0.0016}$ \\
\hline 300 & $\begin{array}{ll} & 0.0024 \\
2 & \\
\end{array}$ & $34^{0.002}$ & $\begin{array}{ll} & 0.0023 \\
3 & \\
\end{array}$ \\
\hline 400 & $\begin{array}{l}8^{0.0023} \\
\end{array}$ & $2^{0.002}$ & $\begin{array}{lr} & 0.0023 \\
1 & \\
\end{array}$ \\
\hline 500 & $8^{0.0032}$ & $13^{0.003}$ & $8^{0.0030}$ \\
\hline 600 & $6^{0.0034}$ & $\begin{array}{r}0.003 \\
35 \\
\end{array}$ & $3 \begin{array}{l}0.0032 \\
3\end{array}$ \\
\hline
\end{tabular}




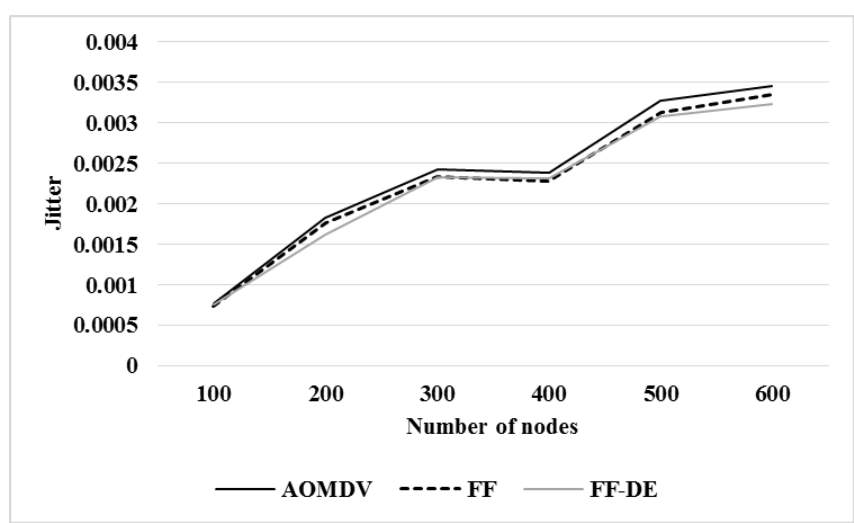

Fig 5 Jitter for FF-DE

From the Fig 5, it can be observed that the FF-DE has lower jitter by $1.31 \% \& 2.66 \%$ for 100 number of nodes, by $12.17 \% \& 8.84 \%$ for 200 number of nodes, by $3.78 \%$ \& $0.42 \%$ for 300 number of nodes, by $2.98 \% \& 1.31 \%$ for 400 number of nodes, by $6.28 \% \& 1.61 \%$ for 500 number of nodes and by $6.87 \%$ \& $3.64 \%$ for 600 number of nodes when compared with AOMDV and FF.

Table V Normalized Routing Load for FF-DE

\begin{tabular}{|c|c|c|c|}
\hline Number of nodes & AOMDV & FF & FF-DE \\
\hline 100 & 0.19 & 0.18 & 0.18 \\
\hline 200 & 0.2 & 0.19 & 0.18 \\
\hline 300 & 0.24 & 0.23 & 0.22 \\
\hline 400 & 0.36 & 0.35 & 0.33 \\
\hline 500 & 0.37 & 0.35 & 0.34 \\
\hline 600 & 0.4 & 0.39 & 0.35 \\
\hline
\end{tabular}

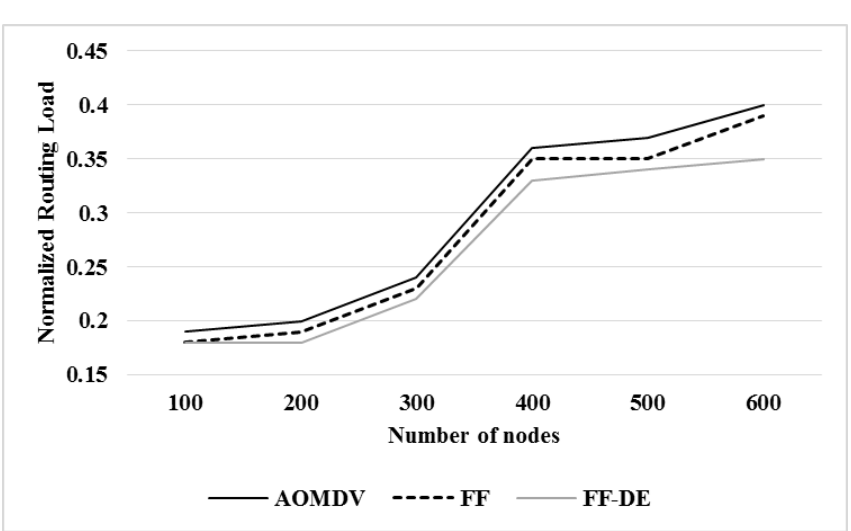

Fig 6 Normalized Routing Load for FF-DE

From the Fig 6, it can be observed that the FF-DE has lower normalized routing load by $5.41 \%$ \& same value for 100 number of nodes, by $10.52 \% \& 5.41 \%$ for 200 number of nodes, by $8.69 \%$ \& $4.44 \%$ for 300 number of nodes, by $8.69 \%$ $\& 5.88 \%$ for 400 number of nodes, by $8.45 \%$ \& $2.89 \%$ for 500 number of nodes and by $13.33 \% \& 10.81 \%$ for 600 number of nodes when compared with AOMDV and FF.

\section{CONCLUSION}

The MANETs are rapidly deployable and self-organizing without a fixed infrastructure. Choosing an optimal path may be challenging. The work proposed another new mechanism for the selection of route with the AOMDV protocol and the hybrid FF along with the DE algorithm for improving QoS. This AOMDV protocol was an extension of the AODV to compute link disjoint paths and the loop-free paths. The FF is inspired by nature and has its roots in the process of attraction of light intensity of the fireflies. The DE, on the other hand, is the evolutionary algorithm employing mutation, recombination and selection. The FF and the DE are very powerful and effective algorithms. The FF, however, is dependent on the random directions of search resulting in retardation and in identifying the best solution. The DE requires more iteration in finding a proper solution. As a result, this proposed method has been designed to cover each algorithm deficiencies so as to make them more suitable for optimization in real world domain. Results show that the FF-DE has higher average PDR by $7.92 \% \& 6.43 \%$ for 100 number of nodes, by $9.78 \% \& 8.21 \%$ for 200 number of nodes, by $7.28 \%$ \& $5.52 \%$ for 300 number of nodes, by $9.8 \%$ $\& 8.56 \%$ for 400 number of nodes, by $8.77 \%$ \& $7.55 \%$ for 500 number of nodes and by $8.01 \% \& 6.32 \%$ for 600 number of nodes when compared with AOMDV and FF.

\section{REFERENCES}

1. P., Periyasamy, \& E. Karthikeyan, (2014). A Novel Approach To Enhance The Quality Of AOMDV Routing Protocol For Mobile Ad Hoc Networks. Journal of Theoretical \& Applied Information Technology, 69(2), 394-404.

2. S., Prabha, \& R. Yadav, (2018, February). Optimal Path in Mobile Ad-hoc Networks with Differential Evolution. In 2018 Recent Advances on Engineering, Technology and Computational Sciences (RAETCS) (pp. 1-5). IEEE.

3. P. K., Manohari, \& N. Ray, (2016, February). Multipath routing protocols in MANETs: A study. In Innovation and Challenges in Cyber Security (ICICCS-INBUSH), 2016 International Conference on (pp 91-96). IEEE.

4. C. V. R. G. N., Satish, \& P. S. V. K. Kumar, (2013). Challenges and Advances in QoS Routing Protocols for Mobile Ad Hoc Networks. International Journal, 3(8).

5. O.S., Badarneh, \& M. Kadoch, (2009). Multicast routing protocols in mobile ad hoc networks: A comparative survey and taxonomy. EURASIP Journal on Wireless Communications and Networking, 2009, 26.

6. K., Kaur, \& L. Pawar, (2015). Review of Various Optimization techniques in MANET Routing Protocol. International Journal of Science, Engineering and Technology Research (IJSETR), 4(8).

7. S., Prabha, \& R. Yadav, (2019). Trusted-Differential Evolution Algorithm for Mobile Ad Hoc Networks. In Recent Trends in Communication, Computing, and Electronics (pp. 181-193). Springer, Singapore.

8. R., Chaudhry, S., Tapaswi, \& N. Kumar, (2018). Forwarding Zone enabled PSO routing with Network lifetime maximization in MANET. Applied Intelligence, 1-28.

9. S., Labed, A., Kout, S., Chikhi, \& E.B. Bourennane, (2018). A hybrid bio-inspired approach to solving the routing problem in mobile ad hoc networks. International Journal of Systems, Control and Communications, 9(3), 185-209.

10. S., Jaiswal, \& N. Kaur, (2018). Energy Efficient and Improved Network Lifetime Multipath Routing using FF-AOMDV and Dragonfly Topology. Energy, 7(15), 7-14.

11. S., Prabha, \& R. Yadav, (2018, February). Optimal Path in Mobile Ad-hoc Networks with Differential Evolution. In 2018 Recent Advances on Engineering, Technology and Computational Sciences (RAETCS) (pp. 1-5). IEEE.

12. W., Wei, Y., Qin, \& Z. Cai, (2018). A multi-objective multicast routing optimization based on differential evolution in MANET. International Journal of Intelligent Computing and Cybernetics, 11(1), 121-140.

13. M., Vijayalakshmi, \& D.S. Rao, (2018). QoS aware multicasting using the enhanced differential evolution cuckoo search routing protocol in MANET. International Journal of Mobile Network Design and Innovation, 8(4), 215-224. 
14. S., Singh, M., Koslia, \& R.C. Poonia, (2018). A GA-QMR: Genetic Algorithm Oriented MANET QoS Multicast Routing. Recent Patents on Computer Science, 11(4), 268-275.

15. R., Kondaiah, \& B. Sathyanarayana, (2018). Trust based Genetic Neuro-Fuzzy System for Intrusion Detection and Self Adaptive Firefly integrated Particle Swarm Optimization Algorithm for Secure Routing in MANET. International Journal of Applied Engineering Research, 13(8), 5722-5735.

16. D., Sarkar, S., Choudhury, \& A. Majumder, (2018). Enhanced-Ant-AODV for optimal route selection in mobile ad-hoc network. Journal of King Saud University-Computer and Information Sciences.

17. S., Mueller, R.P., Tsang, \& D. Ghosal, (2004). Multipath routing in mobile ad hoc networks: Issues and challenges. In Performance tools and applications to networked systems (pp. 209-234). Springer, Berlin, Heidelberg.

18. P., Dahiya, G., Madan, \& R. Gupta, (2014). Performance evaluation of AODV and AOMDV on the basis of throughput. International Journal of Computer Science and Mobile Computing, 3(9), 277-283

19. X.S. Yang, (2010). Firefly algorithm, stochastic test functions and design optimisation. arXiv preprint arXiv:1003.1409.

20. I.B. Aydilek, (2018). A hybrid firefly and particle swarm optimization algorithm for computationally expensive numerical problems. Applied Soft Computing, 66, 232-249.

21. Y.C., Wu, W.P., Lee, \& C.W. Chien, (2011). Modified the performance of differential evolution algorithm with dual evolution strategy. In International conference on machine learning and computing (Vol. 3, pp. 57-63).

22. L., Zhang, L., Liu, X.S., Yang, \& Y. Dai, (2016). A novel hybrid firefly algorithm for global optimization. PloS one, 11(9), e0163230.

23. S., Sarbazfard, \& A. Jafarian (2016). A Hybrid Algorithm Based on Firefly Algorithm and Differential Evolution for Global Optimization. International Journal of Advanced Computer Science and Applications, 7(6), 95-106.. 